\title{
Proopiomelanocortin Expression in both GABA and Glutamate Neurons
}

\author{
Shane T. Hentges, ${ }^{1}$ Veronica Otero-Corchon, ${ }^{2}$ Reagan L. Pennock, ${ }^{1}$ Connie M. King, ${ }^{1}$ and Malcolm J. Low ${ }^{2,3}$ \\ ${ }^{1}$ Department of Biomedical Sciences, Colorado State University, Fort Collins, Colorado 80523, and ${ }^{2}$ Center for the Study of Weight Regulation and \\ ${ }^{3}$ Department of Behavioral Neuroscience, Oregon Health \& Science University, Portland, Oregon 97239
}

\begin{abstract}
Proopiomelanocortin (POMC) neurons have been intensively studied because of their essential role in regulating energy balance and body weight. Many effects of POMC neurons can be attributed to their release of cognate neuropeptides from secretory granules in axon terminals. However, these neurons also synaptically release non-peptide neurotransmitters. The aim of this study was to settle the controversy whether there are separate populations of POMC neurons that release GABA or glutamate. Transgenic mice expressing a red fluorescent protein [Discosoma red (DsRed)] driven by Pomc neuronal regulatory elements (POMC-DsRed) were crossed to mice that expressed green fluorescent protein (gfp) in GABAergic neurons (GAD67-gfp). Approximately 40\% of POMC neurons in the arcuate nucleus of the double-transgenic mice expressed the GAD67-gfp transgene. In vitro neurotransmitter release was detected using wholecell electrophysiologic recordings in cultured GAD67-gfp-positive and GAD67-gfp-negative P0MC neurons that had formed recurrent synapses (autapses). Autapses from GAD67-gfp-positive neurons were uniformly GABAergic. In contrast, autapses from the GAD67gfp-negative POMC neurons exclusively exhibited postsynaptic currents mediated by glutamate. Together, these results indicate that there are two subpopulations of POMC neurons in the arcuate nucleus differentiated by their amino acid neurotransmitter phenotype. Whole-cell voltage-clamp recordings from POMC neurons in live brain slices indicated that GABAergic and glutamatergic POMC neurons are under similar presynaptic and postsynaptic regulation, although the GABAergic POMC neurons are smaller and have higher input resistance. GABAergic and glutamatergic POMC neurons may mediate distinct aspects of POMC neuron function, including the regulation of energy homeostasis.
\end{abstract}

\section{Introduction}

Proopiomelanocortin (POMC) neurons are a critical component of the circuitry that regulates food intake and energy balance (Coll, 2007). These neurons express POMC peptides, including $\alpha$-melanocyte-stimulating hormone (MSH) and $\beta$-endorphin, and project broadly to subcortical forebrain, midbrain, and brainstem nuclei (Jacobowitz and O'Donohue, 1978; Eskay et al., 1979; Chronwall, 1985). Mutations that interfere with the release or signaling of POMC peptides have profound effects on metabolic homeostasis (Coll et al., 2004). POMC neurons also express transmitters that are not derived from the Pomc gene, including endocannabinoids (Vrang et al., 1999; Hentges et al., 2005), cocaine- and amphetamine-regulated transcript (Vrang et al.,

Received Aug. 3, 2009; revised Aug. 27, 2009; accepted Sept. 3, 2009.

This work was funded by National Institutes of Health Grants DK078749 and DK062219 (S.T.H.) and DK066604 (M.J.L.). The GAD67- gfp transgenic mice and tdimer2(12) DsRed plasmid were generously provided by Drs. Yuchio Yanagawa and Roger Tsien, respectively. We thank the Oregon Health \& Science University Transgenic Core Laboratory for pronuclear microinjection of the POMC-DsRed transgene and Dr. John T. Williams for providing helpful insight on the experiments and this manuscript.

Dr. Low is the inventor of technology licensed to Orexigen Therapeutics Inc., a company that may have a commercial interest in the results of this research and technology. This potential conflict of interest has been reviewed and managed by the Oregon Health \& Science University Conflict of Interest in Research Committee.

Correspondence should be addressed to Shane T. Hentges, Colorado State University, Department of Biomedical Science-Neuroscience, 1617 Campus Delivery, Fort Collins, $C 0$ 80523-1617. E-mail: hentgess@colostate.edu.

V. Otero-Corchon's and M. J. Low's present address: Department of Molecular and Integrative Physiology, University of Michigan School of Medicine, Ann Arbor, MI 48109

D01:10.1523/JNEUROSCI.3770-09.2009

Copyright $\odot 2009$ Society for Neuroscience $\quad 0270-6474 / 09 / 2913684-07 \$ 15.00 / 0$
1999), and cholinergic markers (Meister et al., 2006). Although GABA and glutamate are recognized as having important roles in the regulation of energy balance (van den Pol, 2003; Meister, 2007), the colocalization of these transmitters in distinct populations of hypothalamic neurons has just recently begun to be explored.

Conflicting reports exist regarding the amino acid transmitter phenotype of arcuate nucleus POMC neurons. Using transgenic mice with enhanced green fluorescent protein (EGFP)-labeled POMC neurons, it was demonstrated that a substantial proportion of these neurons are immunoreactive for GABA and the vesicular GABA transporter vGAT and express mRNA for the GABA synthetic enzyme GAD (Hentges et al., 2004). It was also found that POMC neurons in primary cultures could release GABA. However, other authors have reported that POMC neurons display immunoreactivity (IR) for the vesicular glutamate transporter vGLUT2, indicating a glutamatergic phenotype (Collin et al., 2003; Kiss et al., 2005). The basis for the differing observations has not been explained previously. Given the important roles that POMC neurons play in regulating energy homeostasis, it is necessary to have a clear understanding of the transmitter phenotype of these neurons. Therefore, the aim of the present study was to determine whether separate groups of POMC neurons could be defined based on whether they store and release GABA or glutamate from their presynaptic terminals. 

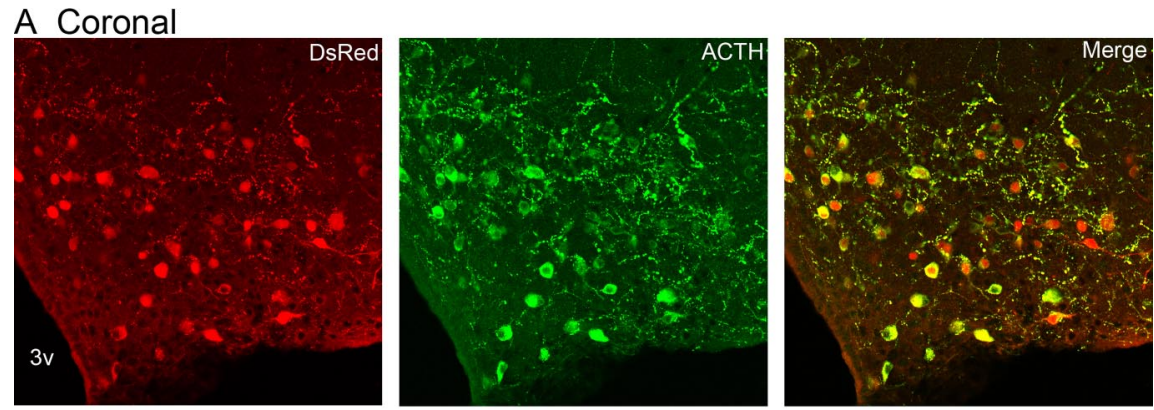

\section{B Sagittal}
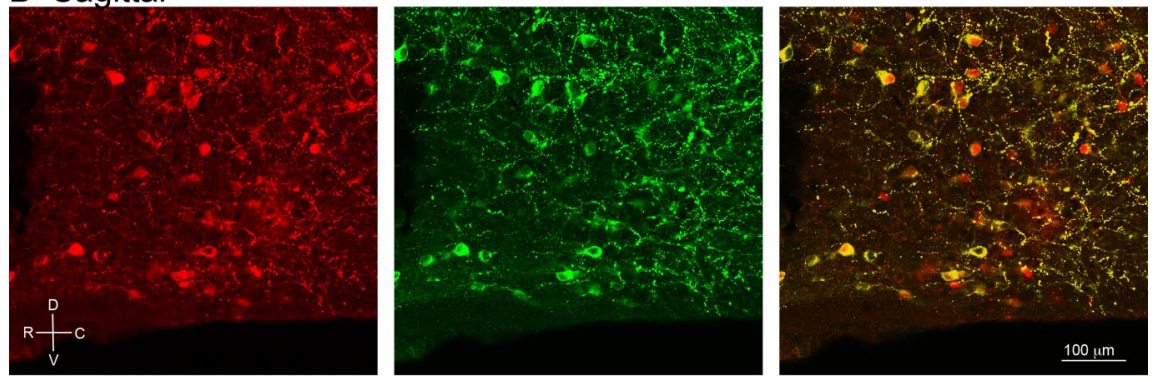

Figure 1. Colocalization of the POMC-DsRed fluorophore and immunoreactivity for ACTH. The Distribution of POMC neurons is indicated by the presence of DsRed in coronal $(\boldsymbol{A})$ and sagittal ( $\boldsymbol{B}$; a medial slice cut in the sagittal plane) brain slices from POMC-DsRed transgenic mice (leftmost images). ACTH-immunoreactivity visualized with a FITC-conjugated secondary antibody (middle, green). Right, Merged images show extensive overlap between POMC-DsRed and ACTH immunoreactivity. Rostral (R), caudal (C), dorsal (D), and ventral (V) orientations are indicated in the bottom left.

\section{Materials and Methods}

Animals. Male and female 6- to 10-week-old mice were used in all experiments. Wild-type C57BL/6J mice were purchased from The Jackson Laboratory. Gad67-gfp knock-in mice (Tamamaki et al., 2003) were maintained as heterozygotes congenic on the C57BL/6J background strain. Transgenic mice expressing the fluorescent protein Discosoma red (DsRed) in POMC neurons were produced by standard techniques and backcrossed on the C57BL/6J background. The transgene contained mouse Pomc sequences from $-13.3 /-6.8$ and $-2.1 /+3.2 \mathrm{~kb}$, numbered relative to the transcriptional start site, ending at a SmaI restriction site within the $5^{\prime}$ untranslated region of exon 2 . These regulatory elements, which include the distal 5' neural enhancer elements described previously (de Souza et al., 2005), were ligated to an obligate dimerized form of DsRed [tdimer2(12)] (Campbell et al., 2002), followed by SV40Tantigen poly(A) sequences. Two separate founder lines were initially analyzed (lines 17 and 18). Both had the expected distribution of positive cells based on the known pattern of POMC-expressing cells in the arcuate nucleus. Both founder lines also had expression in the nucleus of the solitary tract and pituitary melanotrophs and corticotrophs. Hippocampal dentate granule cells also expressed the transgene as has been described for other lines of mice with the Pomc promoter driving the expression of a fluorophore (Rubinstein et al., 1993; Gong et al., 2003; Overstreet et al., 2004), although there is no evidence that these neurons express native Pomc. Founder line 18 was used in all of the current studies, and authentic expression of the fluorophore in POMC neurons in the arcuate was determined as described below and demonstrated in Figures 1 and 4.

GAD67-gfp mice were crossed to POMC-DsRed transgenic mice to produce compound transgenic mice with double-labeled POMC and GAD-expressing cells. GFP and DsRed transgenes were detected by PCR in separate reactions using the following sets of primer pairs: forward, $5^{\prime}$ ACCCCGACCACATGAAGCAGC $3^{\prime}$ and reverse, 5' CGTTGGGGTCTTTGCTCAGGG $3^{\prime}$; and forward, 5' TGGCCAACATTGTTCTGCTCCTTG $3^{\prime}$ and reverse, 5' GCACCTTGAAGCGCATGAACTCTT $3^{\prime}$, respectively. Mice were housed under controlled temperatures (22$24^{\circ} \mathrm{C}$ ) and a constant $12 \mathrm{~h}$ light/dark schedule and given standard chow and tap water ad libitum. All experimental procedures met United States
Public Health Service guidelines with the approval of the institutional animal care and use committees.

Immunofluorescence. Mice were anesthetized with $2.5 \%$ ketamine $/ 1 \%$ xylazine $/ 0.5 \%$ acepromazine and perfused transcardially with $10 \%$ sucrose, followed by $4 \%$ paraformaldehyde. Brains were removed and postfixed in $4 \%$ paraformaldehyde overnight at $4^{\circ} \mathrm{C}$. Sagittal and coronal brain sections ( $50 \mu \mathrm{m}$ thick) containing the hypothalamus were prepared on a vibratome. Immunoreactivity for the POMC peptides adrenocorticotropin (ACTH) and/or $\alpha$-MSH was detected in free-floating sections using a rabbit anti-rat ACTH antibody (1:10,000; A. Parlow, National Hormone and Peptide Program, Torrance, CA) or sheep anti$\alpha$-MSH (1:50,000; a gift from J. Tatro, Tufts Medical Center, Boston, MA). The tissue was incubated in $0.3 \%$ Triton X-100 and 3\% normal goat serum in PBS, $\mathrm{pH} 7.4$, followed by incubation in primary antibody overnight at $4^{\circ} \mathrm{C}$ and then washed in PBS. Immunoreactivity was detected with a FITC-conjugated goat anti-rabbit (1:400; Abcam) or a Texas Red donkey-anti-sheep (1:500; Jackson ImmunoResearch) IgG secondary antibody. In POMC-DsRed;GAD67-gfp double-transgenic mice, ACTH-IR was visualized using a goat anti-rabbit secondary antibody conjugated with Alexa-635 (excitation/emission at 633/648 $\mathrm{nm}$, respectively), which was easily distinguished from the DsRed (558/584 nm excitation/emission). $\beta$-Endorphin-IR was detected using a rabbit polyclonal antibody (1:10,000; A. Parlow) and visualized using an Alexa-488-conjugated goat anti-rabbit secondary antibody (1:400; Invitrogen). Imaging was performed on an Olympus FluoView 300 confocal or a Carl Zeiss 510-Meta laser-scanning confocal microscope.

Validation of the ACTH antibody. Two approaches were taken to verify the specificity of the ACTH antibody used in the present study (rabbit anti-ACTH from the National Hormone and Peptide Program, lot \#AFP156102789R). In one set of experiments, the antibody $(1: 10,000)$ was preabsorbed with $10 \mu \mathrm{M}$ of either ACTH 1-39 (product \#H-4998; Bachem) or ACTH 1-24 (product \#H-1150; Bachem) overnight at $4^{\circ} \mathrm{C}$ with gentle agitation before application to the brain sections. Sections exposed to preabsorbed antibody were processed in parallel with sections receiving non-absorbed antibody $(1: 10,000)$. ACTH-IR was detected using a biotinylated goat anti-rabbit secondary antibody. The signal was amplified using the Vectastain $\mathrm{ABC}$ reagents according to the supplied protocol (Vector Laboratories) and visualized with diaminobenzidine $\left(0.25 \mathrm{mg} / \mathrm{ml}\right.$ in Tris-buffered saline with $\left.0.05 \% \mathrm{H}_{2} \mathrm{O}_{2}\right)$.

In a separate set of experiments, immunodetection of ACTH was performed in POMC knock-out mice (Smart et al., 2006) and POMC ${ }^{+/+}$ littermates. Immunodetection was performed as described above with diaminobenzidine as the substrate.

Primary neuron culture. Primary cultures containing POMC neurons were prepared as described previously (Hentges et al., 2004) from hypothalami of 8- to 10-week-old POMC-DsRed;GAD67-gfp doubletransgenic mice. Electrophysiological recordings were made from cells that had been in culture between 10 and $14 \mathrm{~d}$.

Acute slice preparation for electrophysiological recordings. Sagittal slices $(240 \mu \mathrm{m})$ containing the arcuate nucleus were prepared from 6- to 10 week-old male POMC-DsRed;GAD67-gfp double-transgenic mice using a Leica VT1200S vibratome. Slices were prepared in ice-cold Krebs' solution-bicarbonate buffer containing the following (in $\mathrm{mM}$ ): 126 $\mathrm{NaCl}, 2.5 \mathrm{KCl}, 1.2 \mathrm{NaH}_{2} \mathrm{PO}_{4}, 1.2 \mathrm{MgCl}_{2}, 2.4 \mathrm{CaCl}_{2}, 11$ glucose, and 21.4 $\mathrm{NaHCO}_{3} 21.4$ (saturated with $95 \% \mathrm{O}_{2}$ and $5 \% \mathrm{CO}_{2}$ ).

Electrophysiological recordings. Whole-cell recordings in cultured POMC neurons were performed as described previously (Hentges et al., 2004). Cultured cells and acute hypothalamic slices were continuously 
perfused with Krebs' solution-bicarbonate buffer saturated with $95 \% \mathrm{O}_{2}$ and $5 \% \mathrm{CO}_{2}$. Cells expressing GFP and/or DsRed were detected by excitation at $450-485 \mathrm{~nm}$ and viewed with appropriate filters. The internal recording solution contained the following (in $\mathrm{mm}$ ): 57 $\mathrm{KCl}, 70 \mathrm{~K}$-methyl sulfate, $20 \mathrm{NaCl}, 1.5 \mathrm{MgCl}_{2}$, 0.1 EGTA, 10 phosphocreatine, $2 \mathrm{Mg}$-ATP, and 0.5 GTP, buffered with 5 HEPES, pH 7.3. All recordings were made at $37^{\circ} \mathrm{C}$. Patch pipettes had a tip resistance of $1.4-1.8 \mathrm{M} \Omega$ when filled with internal solution. Only cells with an input resistance $>1 \mathrm{G} \Omega$ were used. Drugs were applied by superfusion. Whole-cell recordings were made using an Axopatch-1D or Multiclamp 700B amplifier (Molecular Devices) and collected using AxographX software (Axograph). IPSCs were isolated by blocking glutamate receptors with DNQX and MK801 [(+)-5-methyl-10,11-dihydro-5H-dibenzo [a,d] cyclohepten-5,10-imine maleate] (10 $\mu \mathrm{m}$, Sigma) to block AMPA and NMDA receptors, respectively. Bicuculline methiodide $(10 \mu \mathrm{M}$; Tocris Bioscience) was used to block $\mathrm{GABA}_{\mathrm{A}}$ receptors and isolate EPSCs. EPSCs or IPSCs were detected and counted using AxographX.

Data analysis. All data are presented as mean \pm SEM. Groups of data were compared using Student's $t$ tests. $p<0.05$ was considered significant.

\section{Results}

POMC-DsRed transgene labels

\section{POMC neurons}

Sagittal brain slices from POMC-DsRed transgenic mice were processed for immunofluorescent detection of ACTH. POMCDsRed-expressing neurons throughout the arcuate nucleus displayed immunoreactivity for ACTH (Fig. 1), demonstrating that the POMC-DsRed transgene effectively labels authentic POMC neurons. However, there were a few ACTHimmunoreactive cell bodies with no detectable DsRed. Counting DsRed and ACTH-IR cells from sections from four mice revealed that $89.1 \pm 2.8 \%$ of labeled cells contained both DsRed and ACTH-IR. A total of $6.7 \pm 1.2 \%$ of labeled cells displayed only DsRed and $4.1 \pm 1.2 \%$ had ACTH-IR but no notable DsRed. Thus, the vast majority of POMC-DsRed cells are authentic POMC neurons based on ACTH-IR.

The specificity of the ACTH antibody to a mid- or C-terminal epitope was demonstrated by the lack of staining when the antibody was preabsorbed with ACTH 1-39 peptide (Fig. 2 B) but not by ACTH 1-24 (data not shown) and by the absence of ACTH-IR in POMC knock-out mice (Fig. 2D). Furthermore, the ACTH immunoreactivity localizes to the same population of cells labeled with an $\alpha-\mathrm{MSH}$ antibody, as expected for these two peptide epitopes contained in a single prohormone (Fig. $2 E-G$ ). However, the $\alpha$-MSH-IR was predominately observed in the fibers rather than the somas, which were better visualized with the ACTH antisera. $\beta$-Endorphin-IR was also examined and demonstrated a similar pattern of expression predominately in fibers (data not shown). Thus, although ACTH-, $\alpha$-MSH-, and $\beta$-endorphin-IR are all detected in neurons expressing the POMC prohormone, ACTH-IR was most sensitive for labeling POMC somas.
B Preabsorbed with ACTH 1-39
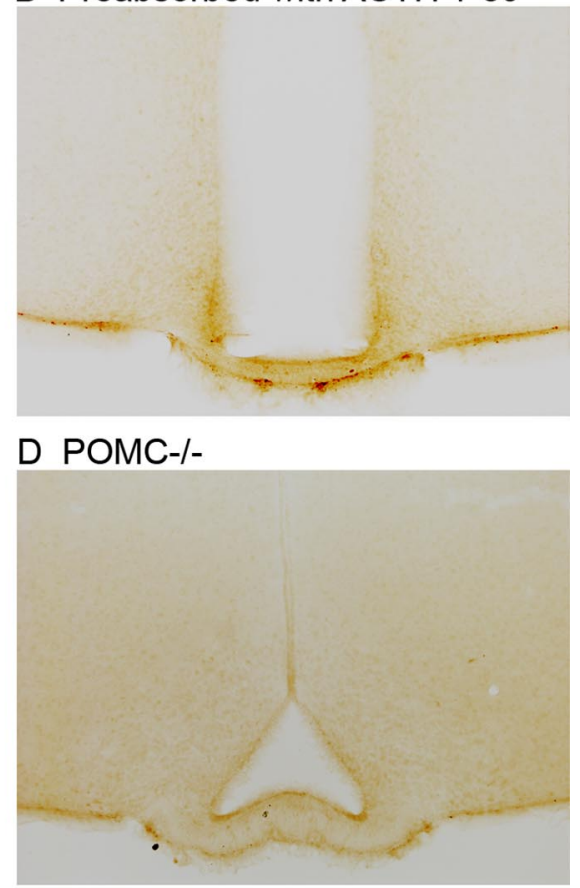

G Merge

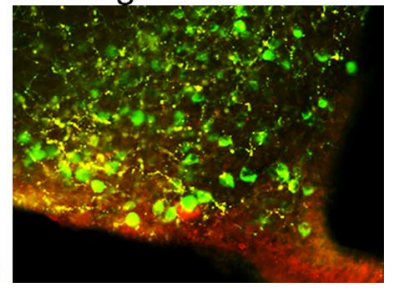

Figure 2. Specificity of the ACTH antibody. A, ACTH-IR in a brain slice containing the arcuate nucleus from a wild-type C57BL/6] mouse visualized with diaminobenzidine and imaged with bright-field microscopy. $\boldsymbol{B}$, Preabsorption of the ACTH antibody with mouse $(\boldsymbol{D})$. Immunofluorescent detection of ACTH $(\boldsymbol{E})$ and $\alpha$-MSH $(\boldsymbol{F})$ in the same brain section shows overlap of the two immunoreactive peptides $(\boldsymbol{G})$, although $\alpha$-MSH-IR is predominately in fibers. R, Rostral; C, caudal; D, dorsal; V, ventral.

\section{GAD67-gfp is expressed in a subset of POMC neurons}

Homozygous POMC-DsRed transgenic mice were crossed to heterozygous GAD67-gfp mice. There were both GAD67-gfppositive and GAD67-gfp-negative POMC neurons in the resulting double-transgenic mice (Fig. 3). The GAD67-gfp transgene was widely expressed throughout the arcuate nucleus and labeled a large number of non-POMC neurons. The proportion of POMCDsRed cells coexpressing GAD67-gfp was determined by counting DsRed-positive cells and DsRed/gfp double-fluorescent cells in eight sections (multiple images from each $50 \mu \mathrm{m}$ section) from each of seven mice (four sectioned sagittal, four coronal). Overall, $42.1 \pm 6.7 \%$ of POMC-DsRed neurons ( $n=7$ mice) expressed the GAD67-gfp transgene. POMC neurons expressing GAD67 were distributed throughout the arcuate nucleus, although a group of POMC neurons lacking GAD67-gfp was always conspicuous in the rostral arcuate nucleus in sagittal sections (Fig. $3 C$, circled). A coronal section through the central arcuate nucleus (approximately bregma $-2.0 \mathrm{~mm}$ ) shows GAD67-positive POMC-DsRed neurons (GABAergic POMC neurons) dispersed throughout the dorsal/ventral and medial/lateral extent of the nucleus (Fig. 3D).

Hypothalamic slices from POMC-DsRed/GAD67-gfp doubletransgenic mice were processed for ACTH-IR to verify that 

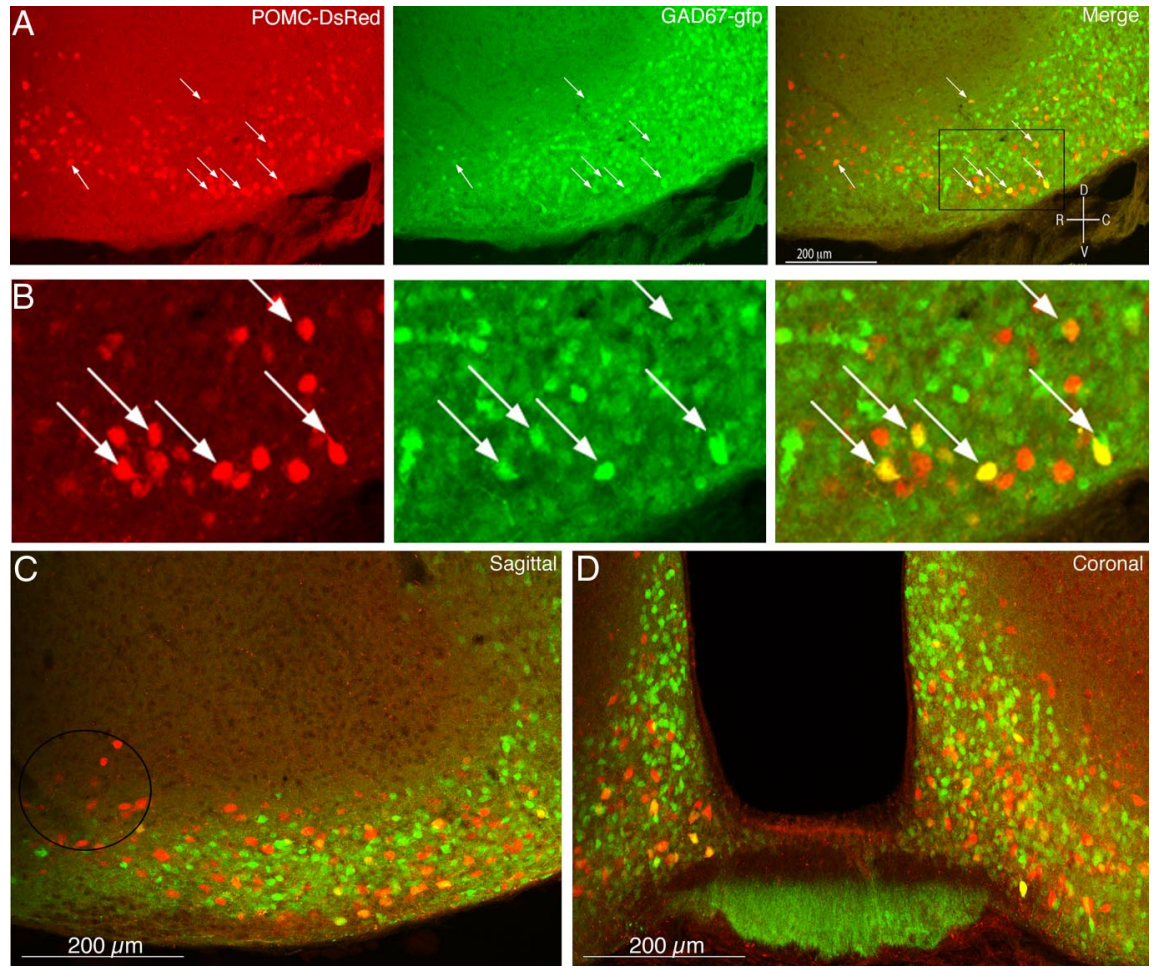

Figure 3. Distribution of GAD-positive and GAD-negative POMC neurons. $A$, Low-power image of a sagittal brain slice containing the arcuate nucleus taken from a POMC-DsRed;GAD67-gfp double-transgenic mouse showing DsRed (left), GAD67-gfp (middle), and a merged image (right). Arrows indicate some of the double-labeled cells. $\boldsymbol{B}$, Larger image of the area outlined by the box in $\boldsymbol{A}$ (right). C, Larger image of a sagittal section from a double-transgenic mouse. The circle indicates a region that is often observed to contain primarily GAD67-gfp-negative POMC-DsRed neurons. D, A coronal section shows double-labeled cells distributed throughout the mediolateral extent of the arcuate nucleus. Orientation for sagittal sections is indicated in the top right. $R$ Rostral; C, caudal; D, dorsal; V, ventral.

both GAD-expressing and GAD-negative POMC-DsRed neurons were authentic POMC neurons. The ACTH-IR was visualized using a secondary antibody labeled with a fluorophore emitting at a higher wavelength than the DsRed so that it was readily distinguished. DsRed-positive neurons expressed ACTH-IR regardless of whether the individual neuron did or did not express GAD67-gfp (Fig. 4). These data are consistent with the observation that nearly all POMC-DsRed neurons displayed ACTH-IR (Figs. 1, 4, $A$ vs $C$ ), although the relative intensity of ACTH-IR and DsRed expression varied among cells. Altogether, the data indicate that a portion of POMC neurons are GABAergic based on the expression of GAD67-gfp.

\section{Characteristics of GABAergic and non-GABAergic POMC neurons in slices}

To determine whether the two subpopulations of POMC neurons may have basal presynaptic or postsynaptic differences, electrophysiological recordings were made to assess basic cellular properties. To examine presynaptic regulation of POMC neurons, spontaneous GABA and glutamate release onto the two types of POMC neurons was detected by recording spontaneous IPSCs (sIPSCs) or sEPSCs, respectively. There was no significant difference in the frequency of sIPSCs $(3.6 \pm 1.3$ vs $5.0 \pm 2.3 \mathrm{~Hz} ; p=$ $0.29 ; n=7-10)$ or $\mathrm{sEPSCs}(0.8 \pm 0.4$ vs $2.07 \pm 1.6 \mathrm{~Hz} ; p=0.19$; $n=7-10)$ recorded in POMC neurons with or without GAD67gfp (Fig. $5 A$ vs $B$, control IPSC traces; summarized in $C$ ). Generally, POMC neurons received a higher frequency of sIPSCs than sEPSCs independent of their GAD67-gfp phenotype (Fig. 5C). Met ${ }^{5}$ Enkephalin (ME) can act through $\mathrm{G}_{\mathrm{i} / \mathrm{o}}$-coupled opioid re- ceptors to inhibit presynaptic transmitter release. Met ${ }^{5}$ Enkephalin $(10 \mu \mathrm{M})$ inhibited IPSCs in GAD67-gfp-positive and GAD67-gfp-negative POMC neurons to the same extent $(52 \pm 15$ and $72 \pm 10 \%$ inhibition, respectively; $p=0.12 ; n=$ 7-10) (Fig. 5D). Altogether, it appears that there is similar presynaptic regulation of all POMC neurons regardless of their expression of GAD67.

Postsynaptic properties were also assessed using whole-cell voltage-clamp recordings (held at $-60 \mathrm{mV}$ ) from GABAergic and GAD67-gfp-negative POMC neurons. POMC neurons express $\mu$-opioid receptors and respond to the peptide ME with a potassium-mediated outward current (Cowley et al., 2001; Hentges et al., 2004; Roseberry et al., 2004). The peak postsynaptic current resulting from bath application of ME (10 $\mu \mathrm{M}$; Sigma) was greater in non-GABA-POMC neurons than the GABA-POMC neurons $(18.3 \pm 2.8$ vs $9.9 \pm 1.9 \mathrm{pA} ; p=0.01 ; n=$ 8-10) (Fig. 6A).

On average, GABA-POMC neurons were smaller and had a higher input resistance than non-GABA-POMC neurons (GAD67-negative) (Fig. 6B,C). The capacitance of GABA-POMC neurons was $8.3 \pm 0.5$ versus $13.7 \pm 0.9 \mathrm{pF}$ for nonGABA-POMC neurons $(p<0.001 ; n=$ 12-15) (Fig. 6C). Accordingly, the input resistance was higher in the small GABAPOMC neurons $(2094 \pm 442 \mathrm{M} \Omega)$ compared with the larger non-GABA-POMC neurons $(1242 \pm 138 \mathrm{M} \Omega ; p=0.04 ; n=$ 14-15) (Fig. 6B).

The larger ME-induced current in non-GABA-POMC neurons can be accounted for by the fact that these neurons were larger than the GABA-POMC neurons. When the ME-induced current was normalized to the capacitance, there was no difference between the amplitude of the ME current in the two groups of POMC neurons (non-GABA-POMC neurons, $1.4 \pm 0.09 \mathrm{pA} /$ pF; GABA-POMC neurons, $1.3 \pm 0.3 \mathrm{pA} / \mathrm{pF} ; p=0.44 ; n=$ $8-10)$. Thus, aside from the presence of GFP, relatively smaller size was the most notable indicator of putative GABAergic POMC neurons.

\section{Release of GABA or glutamate from identified POMC neurons in culture}

The lack of GAD67-gfp in some POMC neurons together with reports that a portion of POMC neurons express the vesicular glutamate transporter-2 (Collin et al., 2003) suggested that the GAD67-gfp-negative POMC neurons may be glutamatergic. To determine the phenotype of non-GABAergic POMC neurons, transmitter release was detected from identified POMC neurons in primary cultures. Primary cultures of hypothalami from POMC-DsRed;GAD67-gfp double-transgenic mice contained both GABAergic POMC neurons and POMC neurons without GAD67 expression (Fig. $7 A, B$, top images). Non-GABA-POMC neurons were observed at a very low percentage $(\sim 20 \%)$ relative to the total number of POMC neurons in culture. When grown at low density in culture, POMC neurons often made recurrent 
synapses, termed autapses. A brief depolarization of the cell body (from -60 to 0 $\mathrm{mV}, 2 \mathrm{~ms}$ ) caused an unclamped action potential in the processes that resulted in the release of transmitter indicated by a postsynaptic current. The postsynaptic currents were identified pharmacologically as IPSCs or EPSCs. Most POMC neurons in primary culture that had formed autapses expressed GAD67-gfp and released GABA as indicated by the recorded IPSC (Fig. 7A, bottom), consistent with our previous report (Hentges et al., 2004). In contrast, all the non-GABAPOMC neurons in these cultures that had formed autapses released glutamate $(n=$ 8) (Fig. $7 B$, bottom). Autaptic currents in every neuron tested were blocked exclusively by either GABA or glutamate receptor antagonists $[10 \mu \mathrm{M}(-)$-bicuculline methiodide or $10 \mu \mathrm{M}$ DNQX, respectively), indicating that GABA and glutamate were not coreleased from the same neuron and that no other currentinducing transmitter was released by the single depolarization protocol. Thus, it appears that distinct populations of POMC neurons release GABA or glutamate.

\section{Discussion}

Both peptide and nonpeptide neurotransmitters are important hypothalamic regulators of food intake and energy balance. To date, there has been an unresolved question whether POMC neurons are GABAergic or glutamatergic (Meister, 2007). The data presented here demonstrate that there are two distinct populations of POMC neurons: one GABAergic and the other glutamatergic. Transgenic labeling and electrophysiologic recording confirm the presence of both inhibitory and excitatory POMC neurons.

\section{GABAergic POMC neurons}

Glutamate decarboxylase is necessary for the production of GABA from glutamate; thus, GAD is a reliable marker for GABAergic neurons (Kaufman et al., 1986; Erlander et al., 1991). Identifying GAD-immunoreactive soma in the arcuate nucleus can be difficult because of the high density of GABAergic neurons in this region and the fact that GAD protein is higher in terminals than in cell bodies, leading to diffuse punctate staining. In the GAD67-gfp transgenic mice, the gfp was highly expressed and distributed throughout the GABAergic cells, making it easy to identify fibers and cell bodies. Furthermore, the transgenic expression permitted electrophysiological recordings from identified GABAergic neurons in live brain slices.

Approximately $35 \%$ of POMC neurons in the mouse arcuate nucleus express Gad mRNA (Hentges et al., 2004). This is consistent with the present finding that $\sim 42 \%$ of POMC-DsRed neurons also express GAD67-gfp. It is possible that GAD67 may not label all GABAergic POMC neurons because some neurons use GAD65 to produce GABA. This possibility cannot be completely ruled out, but mRNA for GAD65 and GAD67 are highly coexpressed in the hypothalamus (Schwartz et al., 1993; Ovesjo et al.,
2001; Meister, 2007). Furthermore, there were no instances in the present study in which GAD67-gfp-negative POMC neurons released GABA from autapses, indicating that the GAD67-gfp transgene efficiently labels GABAergic POMC neurons.

The GABAergic phenotype of POMC neurons was suggested previously by the immunofluorescent detection of the vesicular GABA transporter vGAT and GABA in POMC terminal-like structures (Hentges et al., 2004). Together with the autaptic release of GABA from primary cultures of hypothalamic POMC neurons, it appears highly probable that GABA can be released from POMC terminals in vivo at typical inhibitory synapses.

\section{Glutamatergic POMC neurons}

vGLUTs are necessary to package glutamate for release from presynaptic terminals. Therefore, the presence of vGLUT is used as a marker of glutamatergic neurons. A glutamatergic phenotype for POMC neurons has been suggested previously based on immunodetection of vGLUT2 and in situ hybridization detection of mRNA for vGLUT2 (Collin et al., 2003; Kiss et al., 2005; Meister, 2007). Vesicular glutamate transporters are highly expressed in axon terminals of excitatory neurons. As such, immunodetection of vGLUT yields diffuse punctate staining in the arcuate nucleus (Collin et al., 2003), likely reflecting the fact that this region lacks lamination of fibers.

The possibility that some POMC neurons can release glutamate was further demonstrated here by the fact that POMC neurons devoid of GAD67-gfp released glutamate to mediate an autaptic EPSC. The use of POMC-DsRed;GAD67-gfp double- 
ApomC-DsRed neuron (no GAD67-gfp)

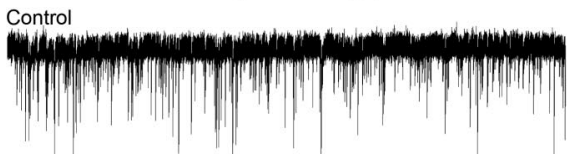

B POMC-DsRed neuron with GAD67-gfp Control

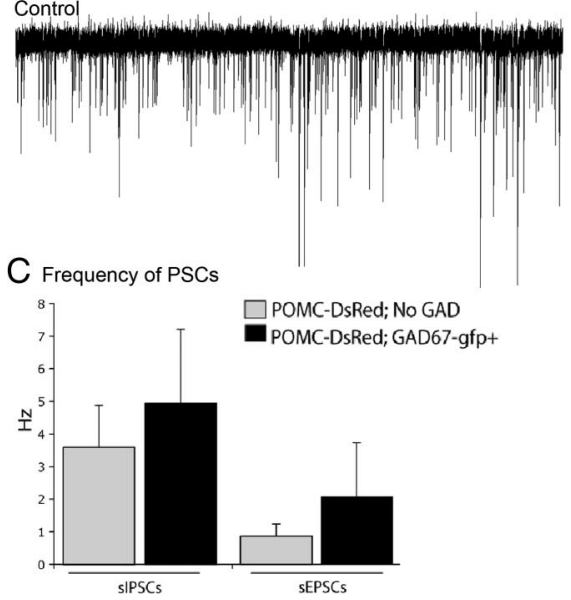

ME $(10 \mu \mathrm{M})$

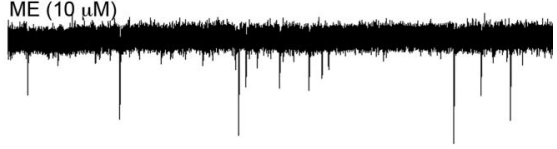

$\mathrm{ME}(10 \mu \mathrm{M})$

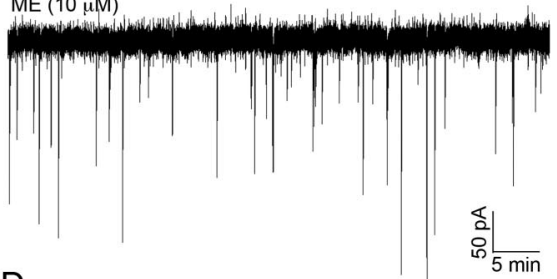

D ME inbition of IPSCs

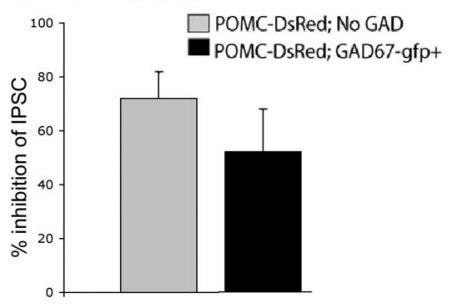

transgenic mice for preparing the primary cultures was an essential step in identifying putative glutamatergic POMC neurons. The previous study using only EGFP-labeled POMC neurons failed to detect any glutamatergic POMC neurons (Hentges et al., 2004). This is likely explained by the observation that only $20 \%$ of all POMC neurons in culture lacked GAD67-gfp expression (and thus are likely glutamatergic) and of those only a small fraction $(<25 \%)$ displayed autaptic currents. It is not clear why there are so few glutamatergic $\mathrm{POMC}$ neurons in the primary cultures when $\sim 60 \%$ of POMC neurons in hypothalamic slices lack GAD67-gfp. The simplest possibility is that the glutamatergic POMC neurons are less resilient to the dissociation and culturing protocol. Alternatively, it could be that GAD67-gfp is not strongly expressed in all GABAergic POMC neurons in vivo and thus the count of GABAergic POMC neurons underestimates the true number.

Figure 5. Presynaptic regulation of GABAergic and non-GABAergic POMC neurons. Spontaneous IPSCs in POMC neurons not expressing GAD67-gfp $(\boldsymbol{A})$ and in GABAergic POMC neurons $(\boldsymbol{B})$ at baseline (left traces) and after a 3-5 min application of ME (right traces). ME-induced inhibition of sIPSCs is summarized in $\boldsymbol{D}(n=7-10)$. C, Frequency of basal sIPSCs and sEPSCs in POMC neurons without GAD $(n=10)$ and with GAD67-gfp $(n=7)$. Error bars represent SEM.

\section{A ME-induced current}

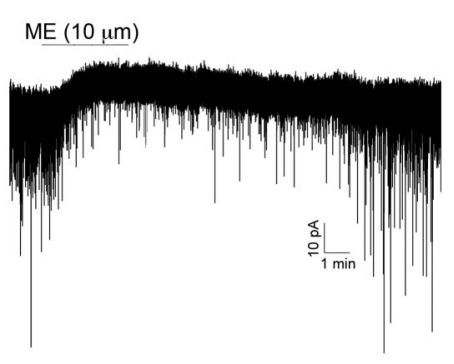

B Input Resistance

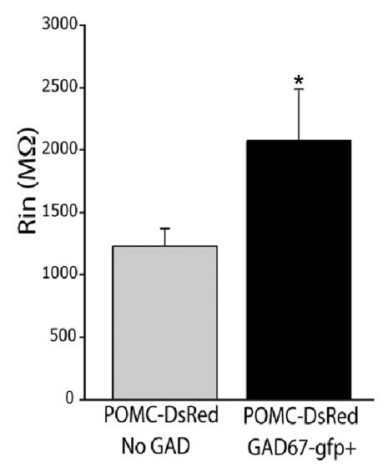

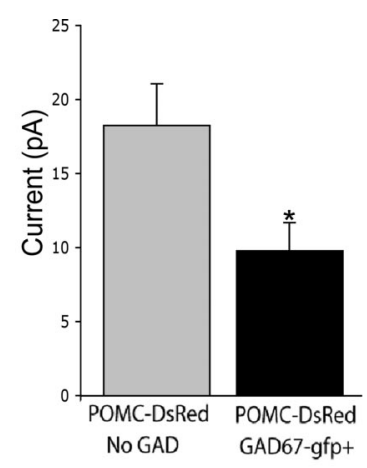

C Capacitance

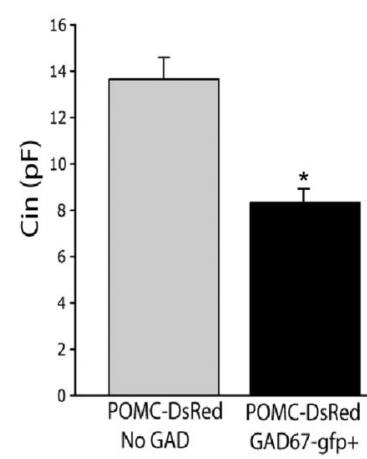

Figure 6. Postsynaptic regulation and properties of GABAergic and non-GABAergic POMC neurons. $A$, Perfusion of ME (10 $\mu \mathrm{M})$ caused an outward current in both GAD67-gfp-positive and GAD67- gfp-negative POMC neurons, although the average magnitude of the current was larger in POMC neurons without GAD $(n=8-10)$. The recording shown in $A$ is a representative trace made in a GABAergic POMC neuron. $\boldsymbol{B}$, Input resistance in GAD-negative (gray bars; $n=14$ ) and GAD67-gfp-expressing (black bars; $n=15$ ) P0MC neurons. C, Capacitance measurements in GAD-negative $(n=12)$ and GAD67-gfp-expressing $(n=15)$ POMC neurons. ${ }^{*} p<0.05$. Error bars represent SEM.

\section{Conclusion}

Although neuropeptides have been heavily studied with regard to energy balance, the release of nonpeptide transmitters from peptidergic neurons is also clearly important for the regulation of energy balance (Tong et al., 2008). The present data demonstrate that POMC neurons in the arcuate nucleus can release either GABA or glutamate. The presence of distinct excitatory and inhibitory subsets of POMC neurons may explain why $\beta$-endorphin has been found previously in terminals making both symmetrical and asymmetrical synapses, which are likely GABAergic and glutamatergic, respectively (Wang and Nakai, 1995; Wang et al., 2001). Given the opposing actions of these two transmitters, it is possible that GABAergic and glutamatergic POMC neurons could have different roles in regulating energy balance. Previously, GABA was found colocalized in POMC terminals in distinct target sites and not others (Hentges et al., 2004), raising the possibility that GABAergic and glutamatergic POMC neurons project to distinct target sites. Overall, the present data describe the presence of a clear and definable heterogeneity among POMC neurons based on the corelease of GABA or glutamate and resolves the apparent discrepancy regarding transmitter phenotype of POMC neurons.

\section{References}

Campbell RE, Tour O, Palmer AE, Steinbach PA, Baird GS, Zacharias DA, Tsien RY (2002) A monomeric red fluorescent protein. Proc Natl Acad Sci U S A 99:7877-7882.

Chronwall BM (1985) Anatomy and physiology of the neuroendocrine arcuate nucleus. Peptides 6 [Suppl 2]:1-11.

Coll AP (2007) Effects of pro-opiomelanocortin (POMC) on food intake and body weight: mechanisms and therapeutic potential? Clin Sci (Lond) 113:171-182.

Coll AP, Farooqi IS, Challis BG, Yeo GS, O’Rahilly S (2004) Proopiomelanocortin and energy balance: insights from human and murine genetics. J Clin Endocrinol Metab 89:2557-2562.

Collin M, Bäckberg M, Ovesjö ML, Fisone G, Edwards RH, Fujiyama F, Meister B (2003) Plasma membrane and vesicular glutamate transporter mRNAs/proteins in hypothalamic neurons that regulate body weight. Eur J Neurosci 18:1265-1278.

Cowley MA, Smart JL, Rubinstein M, Cerdán MG, Diano S, Horvath TL, 
Cone RD, Low MJ (2001) Leptin activates anorexigenic POMC neurons through a neural network in the arcuate nucleus. Nature 411:480-484.

de Souza FS, Santangelo AM, Bumaschny V, Avale ME, Smart JL, Low MJ, Rubinstein M (2005) Identification of neuronal enhancers of the proopiomelanocortin gene by transgenic mouse analysis and phylogenetic footprinting. Mol Cell Biol 25:3076-3086.

Erlander MG, Tillakaratne NJ, Feldblum S, Patel N, Tobin AJ (1991) Two genes encode distinct glutamate decarboxylases. Neuron 7: 91-100.

Eskay RL, Giraud P, Oliver C, Brown-Stein MJ (1979) Distribution of alpha-melanocytestimulating hormone in the rat brain: evidence that alpha-MSH-containing cells in the arcuate region send projections to extrahypothalamic areas. Brain Res 178:55-67.

Gong S, Zheng C, Doughty ML, Losos K, Didkovsky N, Schambra UB, Nowak NJ, Joyner A, Leblanc G, Hatten ME, Heintz N (2003) A gene expression atlas of the central nervous system based on bacterial artificial chromosomes. Nature 425:917-925.

Hentges ST, Nishiyama M, Overstreet LS, StenzelPoore M, Williams JT, Low MJ (2004) GABA release from proopiomelanocortin neurons. J Neurosci 24:1578-1583.

Hentges ST, Low MJ, Williams JT (2005) Differential regulation of synaptic inputs by constitutively released endocannabinoids and exogenous cannabinoids. J Neurosci 25:97469751.

Jacobowitz DM, O’Donohue TL (1978) alphaMelanocyte stimulating hormone: immunohistochemical identification and mapping in neurons of rat brain. Proc Natl Acad Sci U S A 75:6300-6304.

Kaufman DL, McGinnis JF, Krieger NR, Tobin AJ (1986) Brain glutamate decarboxylase cloned in lambda gt-11: fusion protein produces gamma-aminobutyric acid. Science 232:1138-1140.

Kiss J, Csaba Z, Csáki A, Halász B (2005) Glutamatergic innervation of neuropeptide $\mathrm{Y}$ and pro-opiomelanocortin-containing neurons in the hypothalamic arcuate nucleus of the rat. Eur J Neurosci 21:2111-2119.

Meister B (2007) Neurotransmitters in key neurons of the hypothalamus that regulate feeding behavior and body weight. Physiol Behav 92:263-271.

Meister B, Gömüç B, Suarez E, Ishii Y, Dürr K, Gillberg L (2006) Hypothalamic proopiomelanocortin (POMC) neurons have a cholinergic phenotype. Eur J Neurosci 24: 2731-2740.

Overstreet LS, Hentges ST, Bumaschny VF, de Souza FS, Smart JL, Santangelo AM, Low MJ, Westbrook GL, Rubinstein M (2004) A transgenic marker for newly born granule cells in dentate gyrus. J Neurosci 24:3251-3259.

Ovesjö ML, Gamstedt M, Collin M, Meister B (2001) GABAergic nature of hypothalamic leptin target neurones in the ventromedial arcuate nucleus. J Neuroendocrinol 13:505-516.

Roseberry AG, Liu H, Jackson AC, Cai X, Friedman JM (2004) Neuropeptide Y-mediated inhibition of proopiomelanocortin neurons in the arcuate nucleus shows enhanced desensitization in ob/ob mice. Neuron 41:711-722.

Rubinstein M, Mortrud M, Liu B, Low MJ (1993) Rat and mouse proopiomelanocortin gene sequences target tissue-specific expression to the pituitary gland but not to the hypothalamus of transgenic mice. Neuroendocrinology 58:373-380.

Schwartz MW, Sipols AJ, Grubin CE, Baskin DG (1993) Differential effect

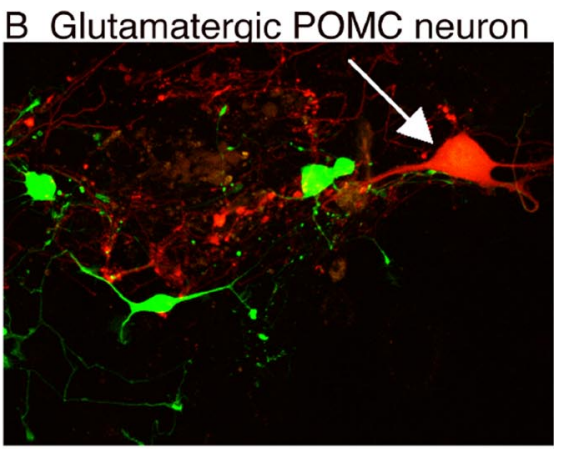

$\mathrm{D}$

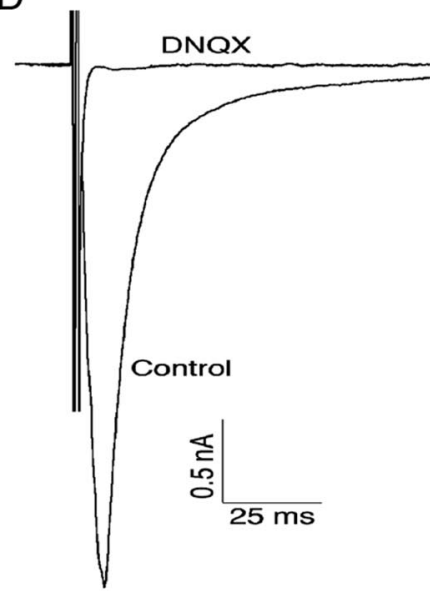

Figure 7. Inhibitory and excitatory POMC neurons. Representative images of a GAD67- gfp-positive POMC-DsRed neuron ( $\boldsymbol{A})$ and a POMC-DsRed neuron that does not express the GAD67-gfp transgene $(\boldsymbol{B})$ in primary neuron cultures. $\boldsymbol{C}$, Depolarization 列 列 GAD67. The current resulting from glutamate release is blocked by the AMPA receptor blocker DNQX. $C$ and $\boldsymbol{D}$ are representative traces from the GABAergic $(n=10)$ and glutamatergic $(n=8)$ cells that displayed autaptic currents.

of fasting on hypothalamic expression of genes encoding neuropeptide $Y$, galanin, and glutamic acid decarboxylase. Brain Res Bull 31:361-367.

Smart JL, Tolle V, Low MJ (2006) Glucocorticoids exacerbate obesity and insulin resistance in neuron-specific proopiomelanocortin-deficient mice. J Clin Invest 116:495-505.

Tamamaki N, Yanagawa Y, Tomioka R, Miyazaki J, Obata K, Kaneko T (2003) Green fluorescent protein expression and colocalization with calretinin, parvalbumin, and somatostatin in the GAD67-GFP knock-in mouse. J Comp Neurol 467:60-79.

Tong Q, Ye CP, Jones JE, Elmquist JK, Lowell BB (2008) Synaptic release of GABA by AgRP neurons is required for normal regulation of energy balance. Nat Neurosci 11:998-1000.

van den Pol AN (2003) Weighing the role of hypothalamic feeding neurotransmitters. Neuron 40:1059-1061.

Vrang N, Larsen PJ, Clausen JT, Kristensen P (1999) Neurochemical characterization of hypothalamic cocaine-amphetamine-regulated transcript neurons. J Neurosci 19:RC5(1-8).

Wang QP, Nakai Y (1995) Immunoelectron microscopy of betaendorphinergic synaptic innervation of nitric oxide synthase immunoreactive neurons in the dorsal raphe nucleus. Brain Res 684: $185-193$.

Wang QP, Guan JL, Shioda S (2001) Immunoelectron microscopic study of beta-endorphinergic synaptic innervation of GABAergic neurons in the dorsal raphe nucleus. Synapse 42:234-241. 